\title{
Keep off-target effects in focus
}

\author{
Concerns about potential unintended DNA changes that might accidentally arise from CRISPR gene editing \\ have emerged to varying degrees with the advent of the technology. As new therapies move from bench to \\ bedside, scientists need to redouble their efforts to document the spectrum of these off-target effects while also \\ acknowledging the reality that a certain degree of risk is embedded in many promising and successful medical \\ therapies.
}

\begin{abstract}
$\mathrm{n}$ the early 1990s when Francisco Mojica was working on his $\mathrm{PhD}$ in the coastal city of Alicante, Spain, he struck upon some genetic sequences in ancient archaea organisms that were curiously repeated up to 600 times in a row. Few people at the time could have predicted that the discovery of
\end{abstract} these repeats, which later became known as clustered regularly interspaced short palindromic repeats (or CRISPR, for short), would set in motion a chain of discoveries over the next quarter-century that would revolutionize gene editing and spur the reimagining of medical treatments. Already, more than 80 patients with cancer in China have received an experimental CRISPR-based therapy, according to a report by The Wall Street Journal. And, as this issue of Nature Medicine went to press, regulators in the United States had given the green light for enrollment in a clinical trial at the University of Pennsylvania using CRISPR gene-editing technology. CRISPR has even arrived in Hollywood. In the major motion picture Rampage, released in April, a crocodile and wolf both ingest CRISPR complexes and become supersized and demonic.

In the film, a geneticist ominously warns that with genetic editing, "changes will be incredibly unpredictable." This runs counter to the idea behind the application of the CRISPR-Cas9 system, which has become a tool of choice to precisely modify DNA because of its targeted and relatively fast action. Yet there is a small grain of truth to the hyperbole of Hollywood: some research has hinted that CRISPR's off-target effects warrant greater attention. Studies of human cells in vitro several years ago suggested that CRISPR-Cas9 could have off-target effects. And some mouse experiments have shown inadvertent DNA changes from CRISPR, producing a quiet drumbeat of worry about off-target effects, particularly with regards to potentially oncogenic mutations, that continues to reverberate in the background as the field marches ahead.

The nucleases used in conjunction with CRISPR create double-stranded breaks, and the notably short guide sequences leading them are not infallible and can cause these breaks to occur at unintended sites in the genome. But concerns about off-target effects resulting from genome editing are by no means confined to CRISPR; these worries have arisen with the arrival of each tool for genetic manipulation. For example, with the advent of viral vector-based gene targeting, researchers cautioned about potentially mutagenic random vector integration into the genome (Nat. Rev. Microbiol. 3, 837-847, 2005). Cell experiments also found hints of possible off-target effects from zinc finger nucleases.

Influenced in part by the movement of CRISPR into clinical testing and a need to monitor any possible unintended biological effects, the US National Institutes of Health announced in January that it had launched the Somatic Cell Genome Editing (SCGE) program. The program will award approximately $\$ 190$ million to researchers over six years to help bring such genomeediting technologies to the clinic and will provide support for improved assays for assessing the potential adverse biological effects of these tools. There are already some assays in hand, including genome-wide unbiased identification of double-stranded breaks enabled by sequencing (GUIDE-seq) and detection of unintended rearrangements by linear amplification-mediated highthroughput genome-wide translocation sequencing (LAM-HTGTS). Seeking to monitor possible unintended DNA alteration due to CRISPR in vivo should remain a priority even as newer CRISPR methods offer a way to edit the genome without making the double-stranded breaks that seem to pose the greatest risk of accidental effects.

A key question is what our increasing knowledge of the off-target effects of CRISPR will mean for the clinical application of these tools, particularly in the eyes of drug regulators. As CRISPR moves ahead, it will be important to observe how off-target effects are captured in different in vivo models and clinical trials. Just because off-target effects are not observed does not mean that they do not exist. Scientists are well versed in the pitfalls of false negatives. Yet even if a therapy carries a certain degree of risk, this should not necessarily disqualify it from the clinic. Some of the most useful medicines employed in the clinic come with mutagenic effects. The chemotherapy drug cisplatin, for example, is a known mutagen but is used to treat a range of malignancies. In some cases, the alternative to treatments such as these can be death, so the risk is tolerated. Clearly the calculus is different for diseases that are less life threatening, and this gets at the heart of medicine.

According to some scientists, the stray mutation frequency for CRISPR using a welldesigned nuclease is thought to fall below the background mutation frequency that occurs spontaneously. So it might be possible for the clinical community to eventually agree that gene editing tools that reach the market should not carry a risk of mutation rates much beyond the frequency of natural spontaneous mutations. Ultimately, regulators and the public should weigh the risks of a therapy against its possible benefits for each disease application. CRISPR is not a magic bullet-it may have off-target effects. But it is also not a shot in the dark.

Published online: 6 August 2018 https://oi.org/10.1038/s41591-018-0150-3 